\title{
Die Anziehung einer Kugel nach Newton
}

\author{
Reinhard Lang \\ Reinhard Lang, geboren 1947, absolvierte das Altsprachliche Gymnasium in Neu- \\ stadt an der Weinstraße und studierte Mathematik und Physik in Heidelberg und \\ Bielefeld. Er promovierte 1976 und habilitierte sich 1983 an der Universität Heidel- \\ berg mit einer Arbeit über Stochastische Modelle von Vielteilchensystemen. Danach \\ war er Heisenbergstipendiat und lehrt und forscht seither am Institut für Angewandte \\ Mathematik der Universität Heidelberg.
}

\section{Einleitung}

Im dreidimensionalen euklidischen Raum seien Punkte $P$ und $S$, in denen Massen $m$ und $M$ konzentriert sind, gegeben. Dem Newtonschen Gravitationsgesetz zufolge ziehen sich die beiden Massen an mit einer Kraft, welche die Richtung der Verbindungslinie $P S$ hat und deren Betrag $F$ durch

$$
F=\gamma \cdot \frac{m M}{P S^{2}}
$$

gegeben ist. Dabei bezeichnet $\gamma$ die Gravitationskonstante und $P S^{2}$ das Quadrat der Entfernung zwischen den Punkten $P$ und $S$. Welches Anziehungsgesetz tritt an die Stelle

Zwischen zwei punktförmigen Massen wirkt eine Gravitationskraft, die laut Newton dem Quadrat ihres Abstandes umgekehrt proportional ist. Ist der Abstand zweier ausgedehnter Massen verglichen mit ihrer Ausdehnung gross, wie dies bei Sonne und Planeten der Fall ist, so ist das Gesetz ganz offensichtlich annäherungsweise erfüllt. Schon Newton stellte sich aber die Frage nach der genauen Formel für die Gravitationskraft einer ausgedehnten Kugel. Seine Theorie sollte ja auch Gravitationserscheinungen in der Nähe der Himmelskörper erklären können, wie etwa Ebbe und Flut. Der rechnerische Nachweis, dass die Gravitationskraft einer homogenen Kugel mit derjenigen einer punktförmigen Masse derselben Grösse übereinstimmt, ist heutzutage eine einfache Übungsaufgabe zum Thema mehrfache Integrale. In seinem Hauptwerk Principia Mathematica bewies Newton diese Tatsache aber mit Hilfe einer geometrischen Überlegung, die allerdings wegen ihrer Kürze nur schwer verständlich ist. - Reinhard Lang rekonstruiert in seinem Beitrag Newtons Beweis und stellt Gemeinsamkeiten fest mit Ideen, die Archimedes zu Volumenberechnungen verwendet hat. ust 
von (1), wenn man anstelle von Punktmassen in $P$ und $S$ homogene Kugeln mit Mittelpunkten $P$ und $S$ und Gesamtmassen $m$ und $M$ hat? Wenn die Kugelradien klein sind gegenüber dem Abstand PS - eine Annahme, die zum Beispiel im Fall von Erde und Sonne zutrifft -, kann man die Kugeln approximativ durch Punktmassen ersetzen. Sofern nur ihr Abstand groß genug ist, ziehen sie sich daher näherungsweise ebenfalls mit der durch (1) gegebenen Kraft an. Die Frage, ob im Fall von Kugeln das Gesetz (1) immer noch gilt, und zwar nicht nur approximativ bei großem Abstand, sondern exakt und für alle Abstände, hat Newton lange Zeit zu schaffen gemacht. In [5], Buch III, Proposition VIII, heißt es:

"After I had found that the force of gravity towards a whole planet did arise from and was compounded of the forces of gravity towards all its parts, and towards every one part was the inverse proportion of the squares of the distances from the part, I was yet in doubt whether that proportion inversely as the square of the distance did accurately hold, or but nearly so, in the total force compounded of so many partial ones; for it might be that the proportion which accurately enough took place in greater distances should be wide of the truth near the surface of the planet, where the distances of the particles are unequal, and their situation dissimilar. But by the help of Prop. LXXV and LXXVI, Book 1, and their Corollaries, I was at last satisfied of the truth of the Proposition, as it now lies before us."

Im Folgenden wird die für das Verständnis des Problems entscheidende Frage nach der Anziehungskraft einer Kugel auf einen außerhalb von ihr gelegenen Massenpunkt betrachtet. Gegeben seien also eine homogene Kugel mit Mittelpunkt in $S$ und Gesamtmasse $M$ und außerhalb der Kugel ein Punkt $P$ der Masse $m$. Die Frage ist, ob die Anziehungskraft exakt durch (1) gegeben ist.

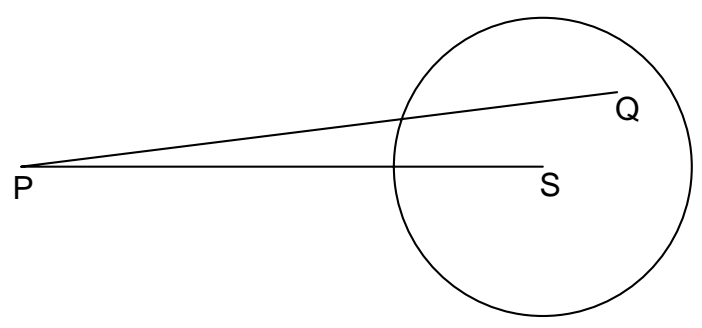

Bild 1

Zunächst ist nicht zu "sehen", welche Kraft aus der Summe der Kräfte zwischen $P$ und den einzelnen Masseteilchen $Q$ innerhalb der Kugel resultiert. Die Lösung dieses Problems war nicht nur hinsichtlich der mathematischen Schönheit der Gravitationstheorie von Interesse, sondern auch im Hinblick auf die experimentelle Bestätigung der Theorie. Will man nämlich die experimentell bekannte Erdbeschleunigung vergleichen mit dem aus der Umlaufzeit des Mondes um die Erde und aus der Entfernung zwischen Erde und Mond berechneten Wert (siehe dazu zum Beispiel [7], p. 152-164), kommt es gerade 
darauf an zu wissen, ob eine Kugel (d.h. die Erde) einen nahe an ihrer Oberfläche befindlichen Massenpunkt (d.h. einen fallenden Körper) nach dem Gravitationsgesetz (1) anzieht.

Vom Standpunkt der heutigen Infinitesimalrechnung aus handelt es sich bei Newtons Frage um die einfache Aufgabe der Berechnung eines mehrfachen Integrals. Da das Problem der Anziehungskraft einer Kugel von großer Bedeutung in der Geschichte der Mathematik und Physik war, ist es jedoch der Mühe wert, die ursprüngliche Lösung aus Newtons Werk selbst heraus zu verstehen zu versuchen. Dies soll im Folgenden geschehen. Newtons Beweis ist elementargeometrisch und umfaßt nur wenige Zeilen, "which must have left its readers in helpless wonder", wie Littlewood in [4], p. 97 schreibt. Littlewood versucht, den Gedankengang von Newtons Beweis zu erklären als die nachträgliche Übersetzung eines zuerst analytisch gefundenen Beweises in eine geometrische Sprache, eine Erklärung, die den Verfasser des vorliegenden Aufsatzes nicht überzeugt. Im Folgenden wird im Gegenteil der Versuch gemacht zu zeigen, daß sich Newtons Beweis fast wie von selbst ergibt, alleine aus der Weise, wie er die Frage gestellt hat: Sein wesentlicher Einfall ist die Formulierung des Problems als eine Suche nach Ähnlichkeiten in gewissen Vergleichsfiguren. Wenn man das Problem einmal so gesehen hat, dann sind die zu seiner Lösung anzustellenden Ähnlichkeitsüberlegungen fast zwangsläufig.

Auch in dem jüngst erschienenen Buch [2] von Chandrasekhar wird Littlewoods Ansicht nicht geteilt. Chandrasekhar erläutert aber den Gang von Newtons Beweis nicht im einzelnen, läßt ihn vielmehr für sich selbst sprechen. Es erscheint daher nicht überflüssig, die folgende genaue Analyse des Beweises mitzuteilen. Diese ist gedacht als eine Fallstudie zum Thema "Einfall und Überlegung in der Mathematik" (vgl. [8]); gleichzeitig könnte sie vielleicht auch dazu dienen, anhand eines speziellen, aber zentralen Problems in das umfassende Werk [2] einzuführen. In [2] werden noch viele weitere schöne Gedanken aus den Principia Mathematica, die im Grenzbereich zwischen Elementargeometrie, Mechanik und Infinitesimalrechnung liegen, behandelt, und das gesamte Werk [2] dürfte daher nicht nur für Historiker, sondern insbesondere auch für Lehrer von großem Interesse sein.

Im nächsten Abschnitt wird erklärt, in welcher Weise Newton das Anziehungsproblem formuliert hat. Daran anschließend wird der sich aus dieser Sicht ergebende Beweisgang heuristisch entwickelt. In Abschnitt 4 folgt dann Newtons Beweis selbst. Ein Vergleich dieses Beweises mit der mechanischen Methode, aufgrund deren Archimedes zum Beispiel das Volumen einer Kugel gefunden hat (vgl. etwa [6], p. 233-238), zeigt erstaunliche Gemeinsamkeiten. Dies wird in Abschnitt 5 dargelegt. Nach diesem Rückblick auf die griechische Mathematik wird im letzten Abschnitt noch ein Ausblick darauf gegeben, welche Bedeutung Newtons Resultat für die weitere Entwicklung der Potentialtheorie hatte, und zwar anhand der von Gauss entdeckten Mittelwerteigenschaft von Potentialen.

\section{Newtons Formulierung der Fragestellung}

Man kann sich die in Bild 1 gezeichnete Vollkugel zerlegt denken in konzentrische Sphären von infinitesimaler Dicke. Die von den einzelnen Sphären auf den Massenpunkt 
$P$ ausgeübten Kräfte addieren sich. Es genügt daher, anstelle einer Vollkugel eine Sphäre zu betrachten. Sie habe die Massendichte 1 und ihr Radius sei $a$. Zu zeigen ist, daß die von der Sphäre auf einen außerhalb von ihr liegenden Massenpunkt $P$ ausgeübte Anziehungskraft dem Betrag nach durch

$$
F_{P}=\gamma \cdot \frac{4 \pi a^{2}}{P S^{2}}
$$

gegeben ist. Zur Bestimmung von $F_{P}$ zerlegt man zweckmäßigerweise die Sphäre in sphärische Zonen von infinitesimaler Breite (vgl. Bild 2).
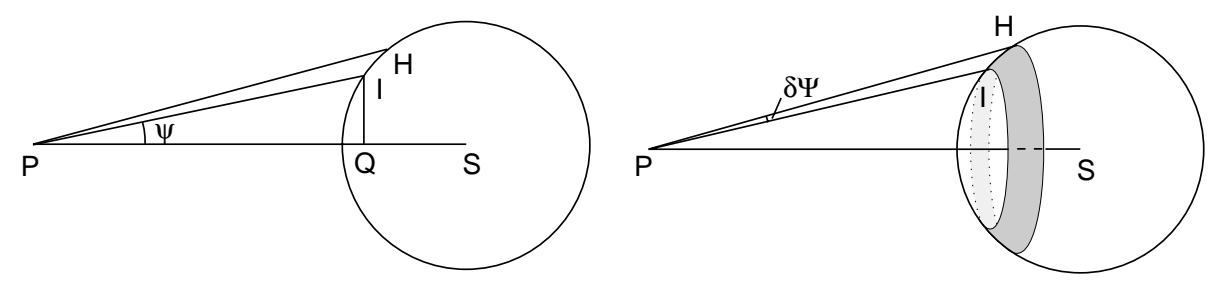

Bild 2

Die Kraft, die von der zum Inkrement $\delta \Psi$ gehörigen sphärischen Zone auf den Punkt $P$ ausgeübt wird, hat aus Symmetriegründen die Richtung $P S$ und ist dem Betrag nach gegeben durch

$$
\delta F_{P}=\left(\frac{1}{P I^{2}} \cos \Psi\right) \cdot(\text { Fläche der Zone })=\left(\frac{1}{P I^{2}} \cos \Psi\right) \cdot(2 \pi I Q \cdot H I) .
$$

Um die Beziehung (2) mit Hilfe von (3) abzuleiten, könnte man zum Beispiel so vorgehen: Man führe $Q S=x$ als Integrationsvariable ein, drücke die auf der rechten Seite von (3) vorkommenden Größen mit Hilfe des Satzes von Pythagoras durch $x$, $d x$ und $P S$ aus und integriere nach $x(-a \leq x \leq+a)$. Das Integral wäre dann mit Hilfe geeigneter Variablensubstitutionen zu berechnen. In dieser Weise könnte man (2) auf einem rechnerischen Weg, den man aber kaum als geometrisch durchsichtig bezeichnen könnte, beweisen.

Tatsächlich ist Newton ganz anders vorgegangen. Um seinen Gedankengang zu verstehen, betrachten wir zunächst die einfachere Frage, wie man aufgrund von Ähnlichkeitsbetrachtungen zeigen kann, daß die von der Sphäre auf einen Punkt $P$ in ihrem Inneren ausgeübte Kraft gleich Null ist. Newton folgend kann man das so sehen wie in Bild 3 gezeigt.

Gegeben $P$ teile man die Sphäre in Paare von gegenüberliegenden quadratischen Flächen $\delta \Sigma$ und $\delta \Sigma^{\prime}$ ein, indem man Sehnen durch den Punkt $P$ zieht, wie in Bild 3 gezeichnet. Die Flächen seien so klein, daß man $\delta \Sigma$ durch $H I^{2}$ bzw. $\delta \Sigma^{\prime}$ durch $K L^{2}$ approximieren kann. Die von $\delta \Sigma$ und $\delta \Sigma^{\prime}$ auf $P$ ausgeübten Kräfte sind dann einander entgegengesetzt 


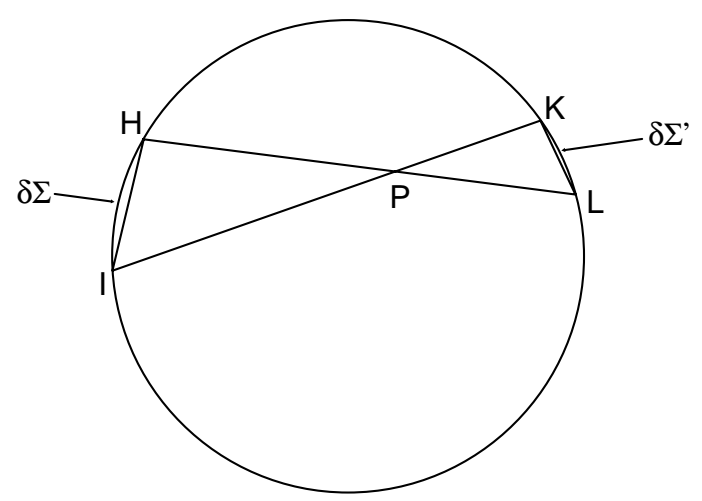

Bild 3

und heben sich gerade auf, denn die Ähnlichkeit der Dreiecke PHI und PKL (die Winkel bei $H$ und bei $K$ sind gleich als Winkel über der gemeinsamen Sehne $I L$ ) impliziert

$$
\frac{\delta \Sigma}{P I^{2}}=\frac{H I^{2}}{P I^{2}}=\left(\frac{H I}{P I}\right)^{2}=\left(\frac{K L}{P L}\right)^{2}=\frac{K L^{2}}{P L^{2}}=\frac{\delta \Sigma^{\prime}}{P L^{2}} .
$$

Dieser Beweis beruht auf zwei Überlegungen:

(a) Aufteilung der Sphäre in geeignete Flächenpaare, mit dem Ziel, eine resultierende Kraft Null zu bekommen.

(b) Eine Ähnlichkeitsbetrachtung, die zeigt, daß sich die von gegenüberliegenden Flächen auf $P$ ausgeübten Kräfte tatsächlich aufheben.

Kann man das Problem der Anziehung eines Punktes im Äußeren der Sphäre verstehen aufgrund von Überlegungen, welche analog zu (a) und (b) sind? An die Stelle des Ziels, eine resultierende Kraft Null zu erhalten, tritt jetzt das Ziel, für alle $P$ außerhalb der Sphäre die Vermutung (2) zu beweisen. Wenn man sich an (a) orientiert, hat man sich zuerst die Frage zu stellen, welche Kräfte man zu diesem Zweck miteinander vergleichen soll. An dieser Stelle kommt Newtons entscheidender Einfall: Er betrachtet zwei Punkte $P$ und $p$ außerhalb der Sphäre und versucht, die von verschiedenen sphärischen Zonen auf $P$ bzw. auf $p$ ausgeübten Kräfte $\delta F_{P}$ bzw. $\delta F_{p}$ miteinander zu vergleichen.
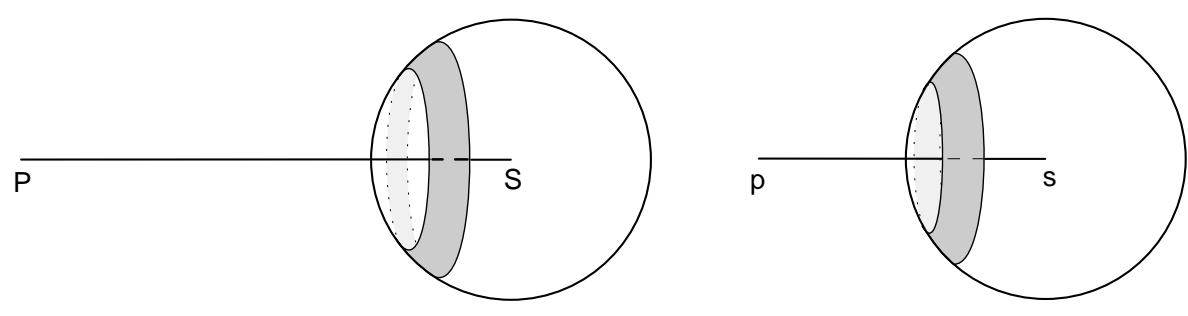

Bild 4a

Bild 4b 
Die Punkte in der Vergleichsfigur (Bild 4b) sind - und werden es auch im Folgenden - mit entsprechenden kleinen Buchstaben bezeichnet. Was die "richtige" Vergleichszone (in Bild 4b schraffiert gezeichnet) ist, die zu einer gegebenen Zone (in Bild 4a schraffiert gezeichnet) paßt, sieht man zunächst nicht. Die gesuchte Zuordnung soll so vorgenommen werden, daß

$$
\frac{\delta F_{p}}{\delta F_{P}}=\frac{P S^{2}}{p s^{2}}
$$

gilt, wobei man, analog zur obigen Überlegung (b), den Nachweis von (5) auf Ähnlichkeitsüberlegungen stützen möchte. Im folgenden Abschnitt wird versucht zu zeigen, wie man, das Ziel (5) vor Augen, auf die "richtige" Zuordnung von Vergleichszonen kommt. Wenn es gelungen ist, bei gegebenen Punkten $P$ und $p$ die Sphäre (Bild 4a) aufzuteilen in disjunkte Zonen $\delta \Sigma_{P}^{(i)}(1 \leq i \leq n)$ und die Sphäre in Bild $4 \mathrm{~b}$ in entsprechende Zonen $\delta \Sigma_{p}^{(i)}$, so daß für die von $\delta \Sigma_{P}^{(i)}$ bzw. $\delta \Sigma_{p}^{(i)}$ auf $P$ bzw. auf $p$ ausgeübten Kräfte $\delta F_{P}^{(i)}$ bzw. $\delta F_{p}^{(i)}$ gilt

$$
\frac{\delta F_{p}^{(i)}}{\delta F_{P}^{(i)}}=\frac{P S^{2}}{p s^{2}}, \quad 1 \leq i \leq n,
$$

folgt daraus die Behauptung (2) unmittelbar. Denn (6) zieht

$$
F_{p}=\sum_{i=1}^{n} \delta F_{p}^{(i)}=\frac{P S^{2}}{p s^{2}} \sum_{i=1}^{n} \delta F_{P}^{(i)}=\left(P S^{2} \cdot F_{P}\right) \cdot \frac{1}{p s^{2}}
$$

nach sich. Bei gegebenem $p$ gilt (7) für alle Punkte $P$ außerhalb der Sphäre. Man kann daher in Formel (7) den Punkt $P$ gegen unendlich streben lassen. Für alle Punkte $Q$ auf der Sphäre läßt sich der Abstand $P Q$ aber abschätzen durch

$$
P S-a \leq P Q \leq P S+a,
$$

so daß wegen (1)

$$
P S^{2} \cdot \gamma \frac{4 \pi a^{2}}{(P S+a)^{2}} \leq P S^{2} \cdot F_{P} \leq P S^{2} \cdot \gamma \frac{4 \pi a^{2}}{(P S-a)^{2}}
$$

folgt. Wenn $P$ gegen unendlich strebt, strebt $P S^{2} \cdot F_{P}$ daher gegen $\gamma \cdot 4 \pi a^{2}$. Eingesetzt in (7) ergibt das gerade die Behauptung (2).

Der springende Punkt des Beweises ist also der Vergleichsansatz (5). Wie ist Newton darauf gekommen? Chandrasekhar ([2], p. 273-275) vermutet, daß es möglicherweise folgendes Problem war, das Newton zum entscheidenden Gedanken geführt hat: Gegeben seien zwei zueinander ähnliche Körper $C_{1}, C_{2}$ und in ähnlicher Lage dazu jeweils ein Punkt $P_{i}$ außerhalb des Körpers $C_{i}(i=1,2)$. In welchem Verhältnis stehen die vom Körper $C_{1}$ bzw. $C_{2}$ auf den Punkt $P_{1}$ bzw. $P_{2}$ ausgeübten Kräfte $F_{1}$ bzw. $F_{2}$ zueinander? Dieses Problem hat Newton mit dem folgenden Ähnlichkeitsargument gelöst ([5], Prop. LXXII einschließlich Cor. III). Zur Vereinfachung der Notation werde angenommen, $C_{i}$ 


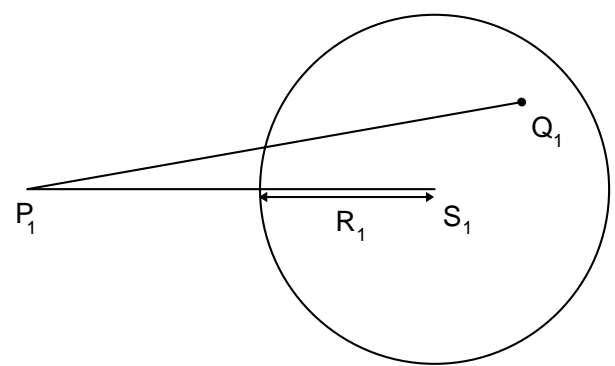

Bild 5a

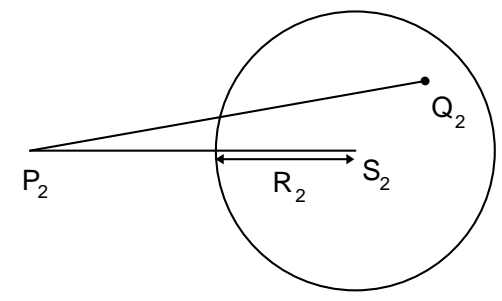

Bild 5b

sei eine Kugel mit Mittelpunkt $S_{i}$ und Radius $R_{i}(i=1,2)$. Nach Voraussetzung gilt für die Punkte $P_{i}$ die Proportion $P_{1} S_{1}: P_{2} S_{2}=R_{1}: R_{2}$.

Die Wahl von passenden "Vergleichsvolumina" liegt in diesem Fall auf der Hand: $\mathrm{Zu}$ gegebenem Punkt $Q_{1}$ in der Kugel $C_{1}$ wähle man $Q_{2}$ so, daß die Dreiecke $S_{1} P_{1} Q_{1}$ und $S_{2} P_{2} Q_{2}$ zueinander ähnlich sind; als infinitesimale Volumenelemente $\delta V_{i}(i=1,2)$ wähle man Würfel mit den Mittelpunkten $Q_{i}$ und Seitenlängen, die sich wie $R_{1}: R_{2}$ zueinander verhalten. Wenn dann $\delta F_{i}$ die von $\delta V_{i}$ auf $P_{i}$ ausgeübte Kraft ist, folgt wegen

$$
P_{1} Q_{1}: P_{2} Q_{2}=P_{1} S_{1}: P_{2} S_{2}=R_{1}: R_{2} \quad \text { und } \quad \delta V_{1}: \delta V_{2}=R_{1}^{3}: R_{2}^{3},
$$

daß sich $\delta F_{1}$ und $\delta F_{2}$ zueinander verhalten wie

$$
\frac{\delta F_{1}}{\delta F_{2}}=\frac{\delta V_{1}}{P_{1} Q_{1}^{2}} \cdot \frac{P_{2} Q_{2}^{2}}{\delta V_{2}}=\frac{\delta V_{1}}{\delta V_{2}} \cdot \frac{P_{2} Q_{2}^{2}}{P_{1} Q_{1}^{2}}=\frac{R_{1}^{3}}{R_{2}^{3}} \cdot \frac{R_{2}^{2}}{R_{1}^{2}}=\frac{R_{1}}{R_{2}} .
$$

Damit ist gezeigt, daß sich die Kräfte $F_{i}$ zueinander verhalten wie die Radien $R_{i}$.

Während die Ähnlichkeit zwischen Bild 5a und Bild 5b von vorneherein gegeben ist, scheinen Bild 4a und Bild 4b durchaus "dissimilar" zu sein. Die in Bild $4 \mathrm{a} / \mathrm{b}$ verborgenen Ähnlichkeiten werden im nächsten Abschnitt ans Licht kommen.

\section{Wie hat man die Vergleichszonen zu wählen?}

In diesem Abschnitt werden heuristische Überlegungen angestellt, um die passenden Vergleichszonen aufzufinden. Gegeben seien also Punkte $P$ und $p$ wie in Bild 4a und in Bild $4 \mathrm{~b}$. Wenn man sich die eingezeichneten sphärischen Zonen parametrisiert denkt durch den Winkel $\Psi$ (vgl. Bild 6), stößt man auf eine erste Schwierigkeit: Der Definitionsbereich von $\Psi$ hängt von der Lage des Punktes $P$ ab.
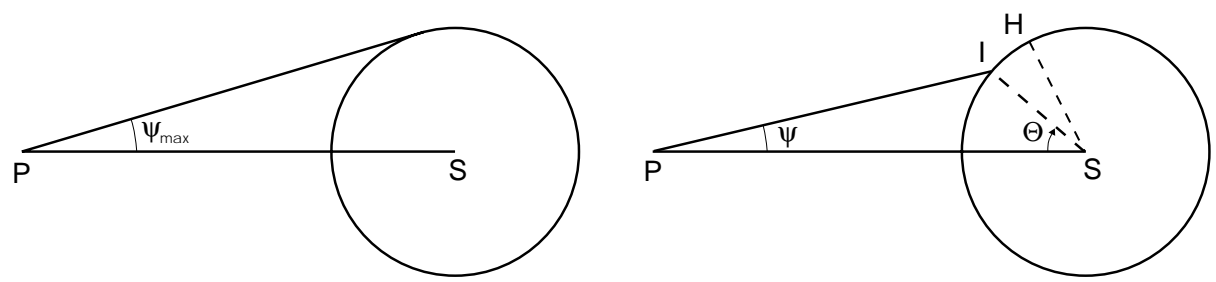

Bild 6 
Es ist daher günstiger, die sphärische Zone $I H$ vom festen Punkt $S$ aus zu betrachten und beispielsweise mit dem Winkel $\Theta$ als Parameter zu arbeiten (vgl. rechtes Bild 6).

Um die richtige Zuordnung zu finden, beginnt man am besten mit der (von der Achse PS aus gezählt) ersten Zone, also einer Kugelkappe (in Bild 7b schraffiert gezeichnet).

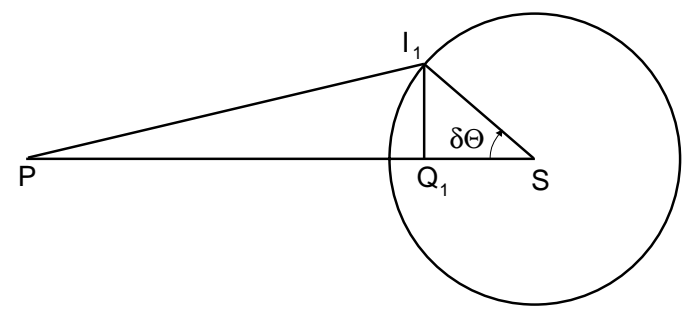

Bild 7a

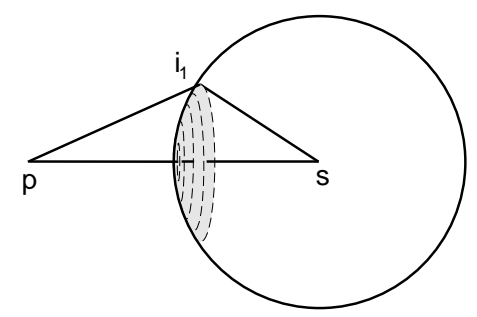

Bild 7b

Sei $I_{1}$ gegeben wie in Bild 7a; der dazu zu findende Vergleichspunkt auf der Vergleichssphäre sei $i_{1}$ (Bild 7b). Die von der Kugelkappe zum Radius $I_{1} Q_{1}$ bzw. zum Radius $i_{1} q_{1}$ auf den Punkt $P$ bzw. $p$ ausgeübte Kraft sei $\delta F_{P}^{(1)}$ bzw. $\delta F_{p}^{(1)}$.
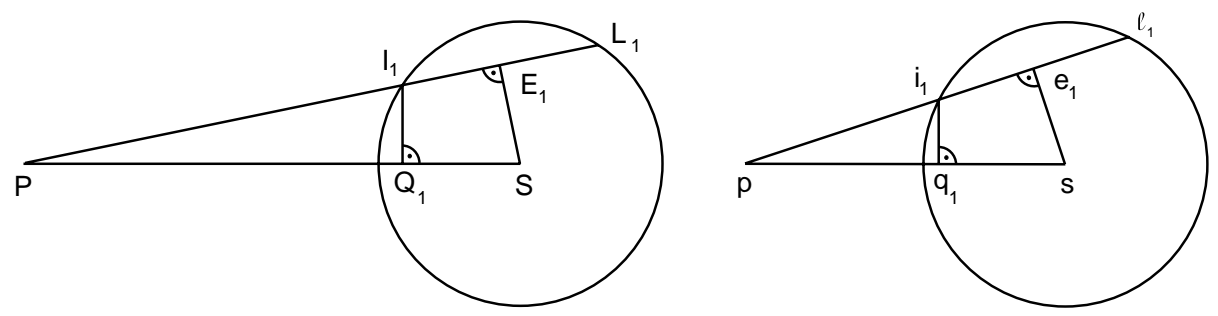

Bild 8

Bis auf einen vernachlässigbaren Fehler ist die Fläche der Kugelkappe gleich der Fläche einer Kreisscheibe mit Radius $I_{1} Q_{1}$ bzw. $i_{1} q_{1}$. Daraus ergibt sich

$$
\frac{\delta F_{p}^{(1)}}{\delta F_{P}^{(1)}}=\frac{P I_{1}^{2}}{I_{1} Q_{1}^{2}} \cdot \frac{i_{1} q_{1}^{2}}{p i_{1}^{2}} .
$$

Wegen

$$
\frac{P I_{1}}{I_{1} Q_{1}}=\frac{P S}{S E_{1}}
$$

ist das Verhältnis (8) genau dann gleich $P S^{2} / p s^{2}$, wenn

$$
s e_{1}=S E_{1}
$$

was äquivalent ist $\mathrm{zu}$

$$
i_{1} \ell_{1}=I_{1} L_{1} .
$$



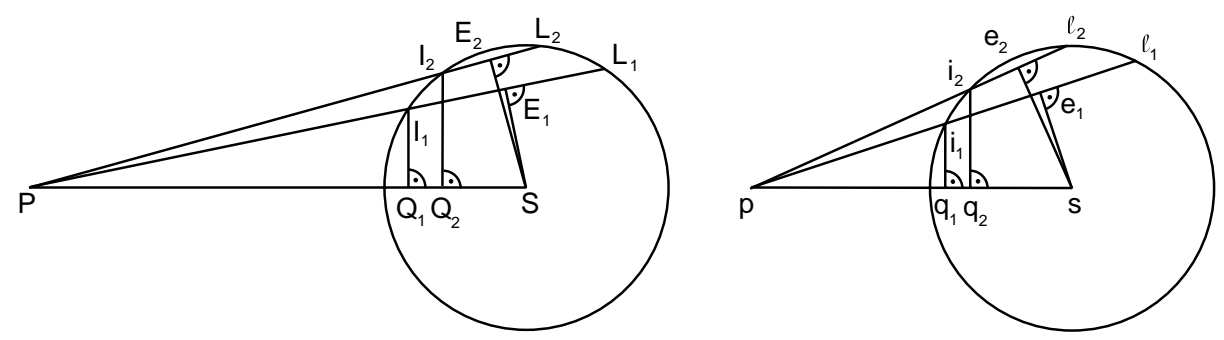

Bild 9

Folglich hat man den Vergleichspunkt $i_{1}$ so festzulegen, daß die Sehnen $i_{1} \ell_{1}$ und $I_{1} L_{1}$ gleich lang sind.

Die an die Kugelkappe anschließende nächste Zone sei durch $I_{1} I_{2}$ gegeben (Bild 9 links). Wie ist der zu $I_{2}$ gehörige Vergleichspunkt $i_{2}$ (Bild 9 rechts) zu wählen? Man versucht, die Wahl analog zur Wahl von $i_{1}$ zu treffen, nämlich so, daß für die zweite Zone die (10) bzw. (11) entsprechende Beziehung zutrifft, das heißt also so, daß

$$
s e_{2}=S E_{2}
$$

und

$$
i_{2} \ell_{2}=I_{2} L_{2}
$$

gilt. Diese Wahl von $i_{2}$ erscheint auch unter dem Gesichtspunkt zweckmäßig, daß dann für die untere Sekante $P L_{2}$ der anschließenden dritten Zone ebenfalls eine Proportion analog zu (9) besteht:

$$
\frac{P I_{2}}{I_{2} Q_{2}}=\frac{P S}{S E_{2}} \text { mit } S E_{2}=s e_{2} .
$$

In dieser Weise legt man die Zuordnung auch der weiteren Zonen fest.

Die so gefundene Zerlegung der beiden Sphären in paarweise einander zugeordnete Zonen kann man auch folgendermaßen beschreiben: Man zeichne in kleinen Abständen voneinander konzentrische Kreise um den Mittelpunkt $S$ bzw. um den Mittelpunkt $s$, wobei die Radien paarweise gleich sind (genau diese Gleichheit ist es, auf der der Beweis von (5) im nächsten Abschnitt aufbauen wird). Dann lege man von den Punkten $P$ bzw. $p$ aus Tangenten an diese Kreise. Die Schnittpunkte mit jeder der beiden Sphären markieren die einander entsprechenden Zonen (Bild 10).
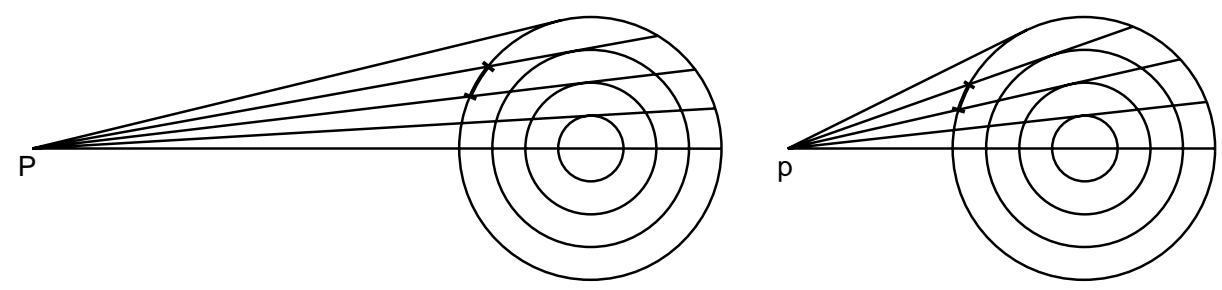

Bild 10 
Hat man die vorangegangene Überlegung mit der Kugelkappe nicht angestellt, erscheint dieser Ansatz verblüffend, vor allem wohl deshalb, weil man sonst nicht so leicht darauf verfällt, die Einteilung der Zonen vom Standpunkt des Sphärenmittelpunktes $S$ aus mittels Tangenten an konzentrische Kreise vorzunehmen. Die Überraschung wird noch größer, wenn man in Bild 10 die konzentrischen Kreise wegläßt.

Im folgenden Abschnitt wird ein Paar der so einander zugeordneten Zonen betrachtet (und zwar solcher Zonen, die auf derjenigen Seite der Sphäre liegen, welche dem Punkt $P$ bzw. $p$ zugewandt ist; für Zonen auf der abgewandten Seite läßt sich der Beweis entsprechend führen), und es wird gezeigt, daß die entsprechenden Kräfte dann auch tatsächlich im Verhältnis (5) zueinander stehen.

\section{Newtons Beweis ("as it now lies before us")}

In Bild 11 sei

$$
s e=S E
$$

und

$$
s d=S D
$$

Die von den Zonen $H I$ bzw. hi auf $P$ bzw. auf $p$ ausgeübten Kräfte seien $\delta F_{P}$ bzw. $\delta F_{p}$.
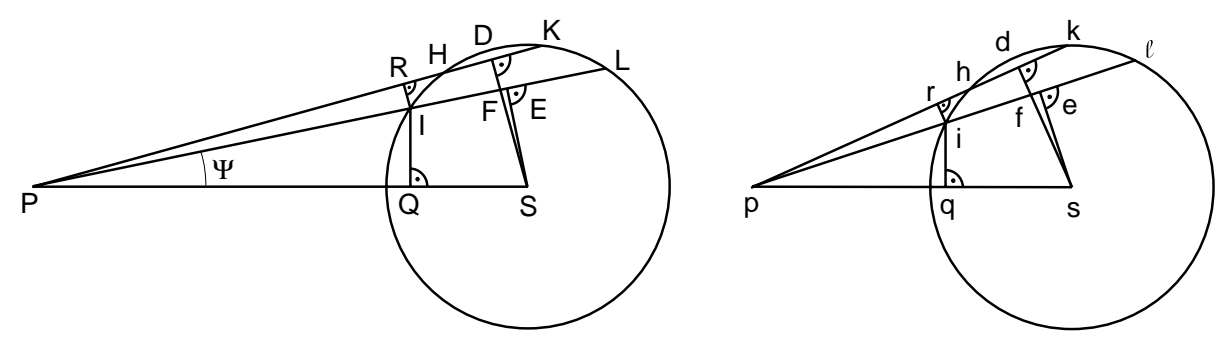

Bild 11

Da der Kosinus des Winkels $\Psi$ bis auf einen vernachlässigbaren Fehler gegeben ist durch $\cos \Psi=P E / P S=P F / P S$, berechnet sich das Verhältnis der Kräfte $\delta F_{P}$ und $\delta F_{p}$ nach (3) $\mathrm{zu}$

$$
\frac{\delta F_{p}}{\delta F_{P}}=\frac{P I^{2}}{p i^{2}} \cdot \frac{P S \cdot p f}{P F \cdot p S} \cdot \frac{h i \cdot i q}{H I \cdot I Q} .
$$

Die Berechnung der rechten Seite von (17) beruht auf zwei Proportionen. Die erste ist Proportion (9) bzw. (14):

$$
\frac{P I}{I Q}=\frac{P S}{S E},
$$

woraus mit (15) folgt

$$
\frac{P I}{I Q} \cdot \frac{i q}{p i}=\frac{P S}{p s}
$$


Die zweite Proportion ergibt sich aus der folgenden Überlegung. In Bild 11 bezeichne $R$ den Fußpunkt des Lotes von $I$ auf die Sekante PK. Aus der Ähnlichkeit der Dreiecke $P R I$ und $P D F$ folgt

$$
\frac{P I}{P F}=\frac{R I}{D F} .
$$

Dem Vorbild (18), (19) folgend möchte man auch diese Proportion mit der entsprechenden für $p i / p f$ verbinden. Analog zu (15) kann man jetzt

$$
D F=d f
$$

benutzen, wobei die Gleichheit approximativ in dem Sinne gilt, daß $D F / d f$ gegen 1 strebt, wenn die Winkel DPF und $d p f$ gemeinsam gegen Null streben. Zur Begründung von (21) benutze man (15) und (16) und approximiere

$$
D F=S D-S F \approx S D-S E=s d-s e \approx s d-s f=d f .
$$

Dabei ist zu beachten, daß die bei dieser Näherung gemachten Fehler wie das Quadrat des Winkels DPF bzw. dpf verschwinden (der Beweis dafür wird am Ende dieses Abschnitts nachgetragen), so daß sie bei der Berechnung des Quotienten $D F / d f$ vernachlässigt werden können. Aus (20) und (21) bekommt man

$$
\frac{P I}{P F} \cdot \frac{p f}{p i}=\frac{R I}{r i}=\frac{H I}{h i},
$$

wobei man die letzte Gleichheit so einsehen kann: Bis auf einen vernachlässigbaren Fehler läßt sich die Gerade durch $H$ und $I$ ersetzen durch die Tangente im Punkt $H$. Nach Voraussetzung (16) ist aber der Winkel zwischen dieser Tangente und der Sekante $P K$ gleich dem Winkel zwischen der Tangente im Punkt $h$ und der Sekante $p k$. Daraus folgt die Ähnlichkeit der Dreiecke RHI und rhi und damit die letzte Proportion in (23).

Jetzt braucht man nur noch die beiden Proportionen (19) und (23) miteinander zu multiplizieren und das Produkt mit (17) zu vergleichen, um das gewünschte Ergebnis

$$
\frac{\delta F_{p}}{\delta F_{P}}=\frac{P S^{2}}{p s^{2}}
$$

$\mathrm{zu}$ erhalten.

Es bleibt noch nachzutragen, weshalb die in (22) gemachten Fehler $S F-S E$ bzw. $s f-s e$ nur von zweiter Ordnung sind. Zum Beweis wendet man den Satz des Pythagoras im Dreieck ESF an und bekommt

$$
S F^{2}=S E^{2}+E F^{2}, \quad S F-S E=E F^{2} /(S F+S E),
$$

d.h. $S F-S E$ ist von der Größenordnung $E F^{2}$. Wegen $\Varangle E S F=\Varangle D P F$ folgt daraus die Behauptung. Damit ist der Beweis von Proportion (5) vollständig. 


\section{Vergleich mit der mechanischen Methode des Archimedes}

Archimedes hat das Volumen einer Kugel mit Hilfe der folgenden heuristischen Methode gefunden (vgl. [1]; [6], p. 233-238; [9], p. 354-357; [8]). Man wählt geeignete Vergleichskörper, deren Volumina bekannt sind, nämlich einen passenden Kegel und einen passenden Zylinder, und hängt Kugel, Kegel und Zylinder an Hebelarmen so auf, daß Gleichgewicht herrscht. Mit Hilfe des Hebelgesetzes läßt sich dann daraus das unbekannte Volumen der Kugel bestimmen. Ähnlich wie bei Newton besteht der wesentliche Einfall darin, passende Vergleichsobjekte zu finden, bei Archimedes im Hinblick auf das Ziel, Gleichgewicht an einem Hebel herzustellen, bei Newton im Hinblick auf das Ziel, das Potenzgesetz (5) zu beweisen. Beide Male kommt es darauf an, Proportionen aufgrund von Ähnlichkeiten zu finden, welche ihrerseits den Weg zum Auffinden der richtigen Vergleichsobjekte gewiesen haben. Sowohl Newton als auch Archimedes sind zu ihrem Vorgehen vermutlich inspiriert worden durch analoge einfachere Probleme, die sie vorher gelöst hatten. Im Fall von Archimedes war es die Aufgabe, die Fläche eines Parabelsegmentes (vgl. Bild 12) zu bestimmen. Im Folgenden soll seine Methode anhand dieses Beispiels demonstriert werden, und zwar so, daß der Leser ihre Ähnlichkeit mit der Vorgehensweise von Newton leicht erkennen kann.

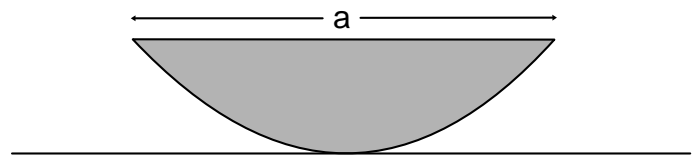

Bild 12

Zur Vereinfachung der Darstellung betrachten wir nur Segmente wie in Bild 12 (Archimedes betrachtet allgemeiner schiefe Segmente) und verwenden kartesische Koordinaten. Archimedes wählt als Vergleichsfigur dasjenige Dreieck, das von den beiden Koordinatenachsen und der Tangente an die Parabel im Punkt $(a, 0)$ begrenzt wird (Bild 13).
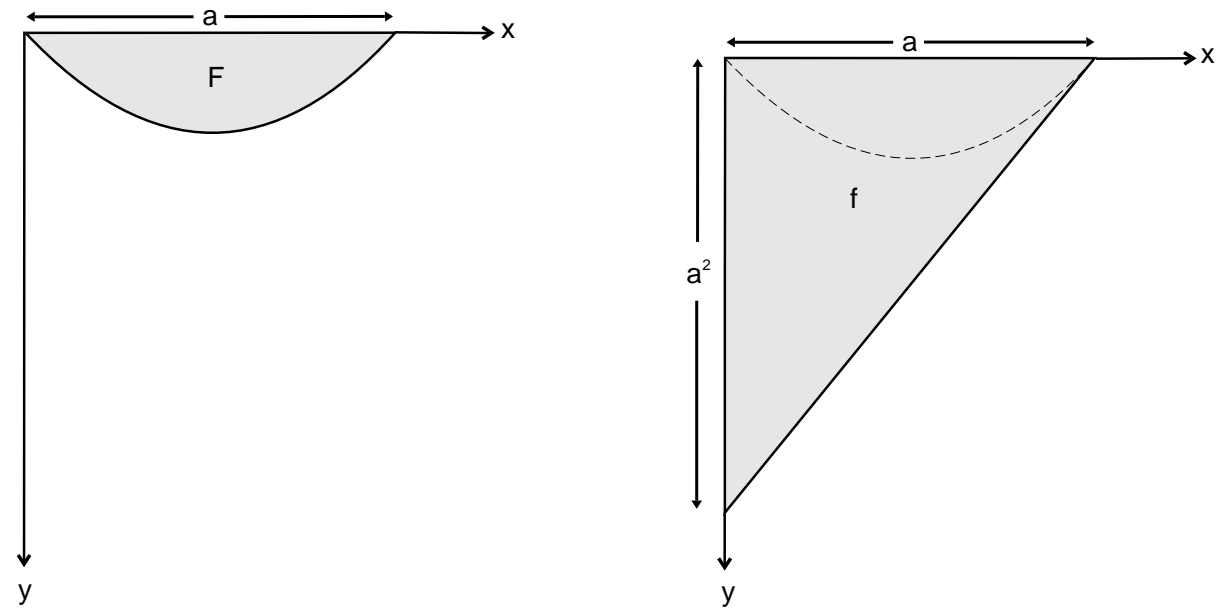

Bild 13 
Mit Hilfe der Hebelmethode soll eine Beziehung zwischen der Fläche $F$ des Parabelsegmentes und der Fläche $f$ des Dreiecks gefunden werden.

Weshalb hat Archimedes gerade dieses Dreieck als Vergleichsfigur gewählt? Der Grund liegt darin, daß zwischen den Ordinaten-Abschnitten von Parabel und Tangente eine einfache Proportion besteht.

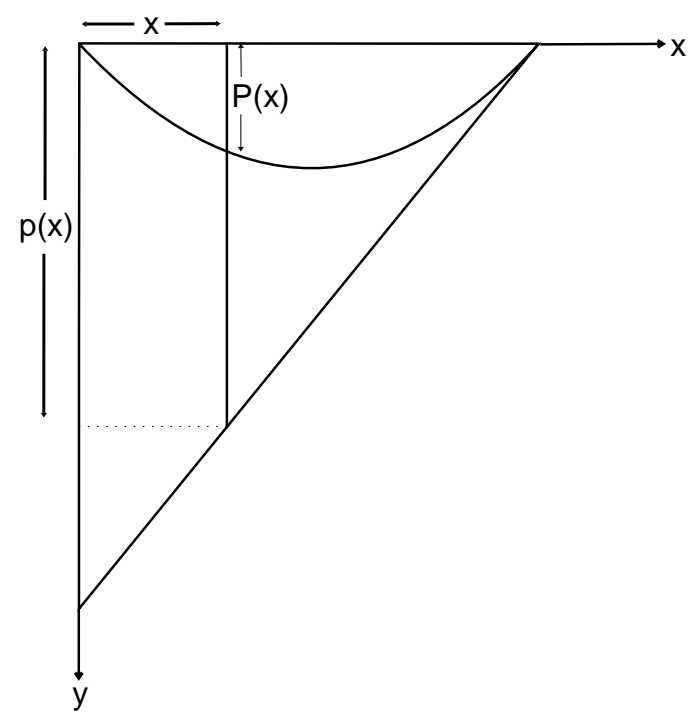

Bild 14

Wenn nämlich $P(x)$ bzw. $p(x)$ die Ordinate der Parabel bzw. der Tangente zur Abszisse $x$ ist $(0 \leq x \leq a)$, so gilt

$$
P(x): p(x)=x: a,
$$

wie unmittelbar aus den Gleichungen

$$
P(x)=x(a-x), \quad p(x)=a(a-x)
$$

abgelesen werden kann (die Tangentengleichung folgt aus $p(a)=P(a)=0$ und $p(x) \geq$ $P(x)$ für alle $x$ wegen $\left.0 \leq(a-x)^{2}=a(a-x)-x(a-x)\right)$. Aufgrund der Proportion (24) kann man das Parabelsegment und das Dreieck folgendermaßen an Hebelarmen so aufhängen, daß Gleichgewicht herrscht: Zunächst denkt man sich beide Figuren zerlegt in infinitesimale Streifen der Höhen $P(x)$ bzw. $p(x), 0 \leq x \leq a$, und hängt diese Streifen an Hebelarme wie in Bild 15.

Da sich die Gesamtfiguren aus den einzelnen Streifen zusammensetzen, bleibt das Gleichgewicht bestehen, wenn man Parabelsegment und Dreieck aufhängt wie in Bild 16.

Man kann sich die Masse des Dreiecks in seinem Schwerpunkt konzentriert denken. Dieser hat die Abszisse $\frac{1}{3} a$ (die durch den Punkt $\left(\frac{a}{2}, 0\right)$ gehende Seitenhalbierende wird 


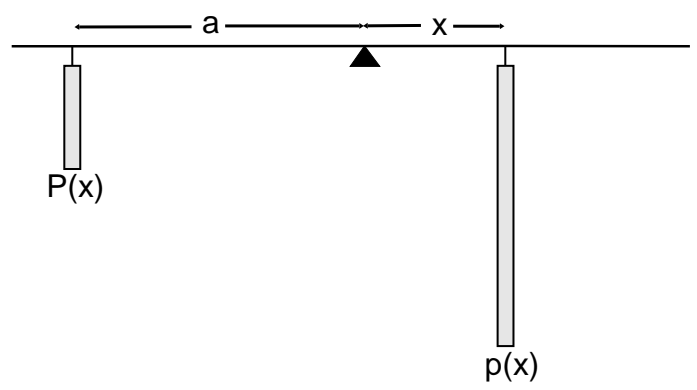

Bild 15

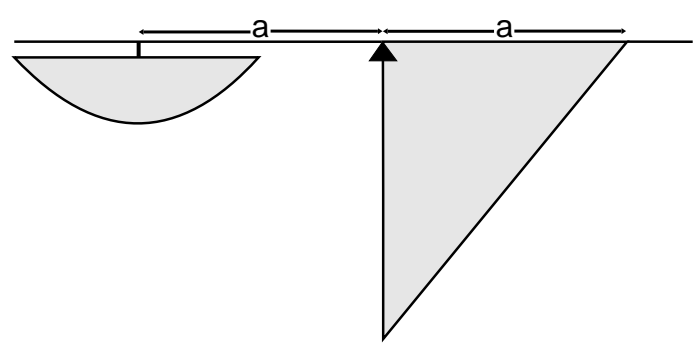

Bild 16

durch den Schwerpunkt im Verhältnis 2:1 geteilt; dessen Abszisse ist also $\frac{2}{3} \cdot \frac{a}{2}$ ). Aus dem Hebelgesetz folgt daher

$$
F: f=\frac{1}{3} a: a,
$$

und die gesuchte Beziehung zwischen den beiden Flächen $F$ und $f$ ist gefunden.

Sowohl bei Archimedes als auch bei Newton werden das Ausgangsobjekt und das Vergleichsobjekt so in infinitesimale Teile zerlegt, daß Proportionen zwischen diesen Teilen bestehen, die im Fall der Parabelquadratur im Hinblick auf das Hebelgesetz angewandt werden, im Fall des Anziehungsproblems im Hinblick auf das zu beweisende Potenzgesetz. Bei Archimedes handelt es sich um ein heuristisches Verfahren zur Auffindung einer unbekannten Fläche oder eines unbekannten Volumens; die Auswahl der Vergleichsfigur ist weitgehend der Phantasie überlassen. Bei Newton ist die Auswahl im wesentlichen schon festgelegt durch die zu beweisende Aussage selbst, welche zuvor vermutet worden war aufgrund der Gravitationstheorie. Im Fall der Hebelmethode könnte man von einem Beispiel physikalischer Mathematik (vgl. [6], Kapitel IX), im Fall von Newtons Ähnlichkeitsbeweis von einem Beispiel mathematischer Physik sprechen.

\section{Newtons Resultat und die Mittelwerteigenschaft}

Wie in [2], p. 10-14 dargelegt, hat Newtons Satz von der Anziehungskraft einer Kugel einen bedeutenden Stellenwert innerhalb des in den Principia Mathematica entwickelten mathematischen Gebäudes. Newtons Beweis ist jedoch auf den Fall der Kugel beschränkt. Die Beweisführung, so einfallsreich und scharfsinnig sie ist, ist von genau jener Weise, 
welche Lagrange bestrebt war, durch allgemeine, auf das gesamte Gebiet der Mechanik anwendbare Methoden zu ersetzen. Im Vorwort zu seiner Analytischen Mechanik (1788) heißt es:

“Les méthodes que j’y expose ne demandent ni constructions, ni raisonnements géometriques ou méchaniques, mais seulement des opérations algébriques, assujetties à une marche régulière et uniforme."

Eine andere Entwicklungslinie, die ebenfalls von Newtons Gravitationstheorie ihren Ausgang genommen hat, ist aus dem Problem entstanden, die Anziehungskraft von allgemeineren Körpern als der Kugel zu bestimmen. Zu diesem Zweck hat es sich als günstig erwiesen, nicht mit der von einer Massenverteilung $\mu$ ausgeübten Gravitationskraft selbst, sondern mit dem zugehörigen Potential $U_{\mu}$, also einer skalaren Funktion, zu arbeiten. Zur Erläuterung formulieren wir zunächst einmal Newtons Resultat von der Anziehungskraft einer Kugel um in Termen von Potentialen. Wenn $\mu$ eine Punktmasse in einem Punkt $y \in \mathbb{R}^{3}$ ist (d.h. im Fall eines Dirac-Maßes $\delta_{y}$ ), ist das zugehörige Potential gegeben durch

$$
U_{\delta_{y}}(x)=-\gamma \cdot \frac{1}{|x-y|}, \quad x \in \mathbb{R}^{3} .
$$

Die bei gegebener Masse $\delta_{y}$ auf einen Massenpunkt an der Stelle $x$ ausgeübte Gravitationskraft $\vec{F}(x)$ erhält man aus $U_{\delta_{y}}$ durch Differenzieren nach $x$ :

$$
\vec{F}(x)=-\operatorname{grad} U_{\delta_{y}}(x)=-\gamma \cdot \frac{x-y}{|x-y|^{3}} ;
$$

insbesondere ist also der Betrag von $\vec{F}(x)$ gegeben durch

$$
|\vec{F}(x)|=\gamma \cdot \frac{1}{|x-y|^{2}}
$$

in Übereinstimmung mit der eingangs in (1) gegebenen Formulierung des Gravitationsgesetzes. Um Newtons Satz mit Hilfe von Potentialen auszudrücken, betrachten wir eine homogen mit Masse belegte Sphäre $S_{r}(x)$ mit Mittelpunkt in $x \in \mathbb{R}^{3}$ und Radius $r>0$. Bei dieser Massenbelegung herrscht an einem Punkt $y \in \mathbb{R}^{3}$ außerhalb der Sphäre dasselbe Potential, als wäre die Gesamtmasse $4 \pi r^{2}$ im Mittelpunkt $x$ konzentriert:

$$
\int_{S_{r}(x)} \sigma(d z) U_{\delta_{z}}(y)=4 \pi r^{2} \cdot U_{\delta_{x}}(y), \quad|y-x|>r,
$$

wobei $\sigma$ das Oberflächenmaß auf $S_{r}(x)$ bezeichnet.

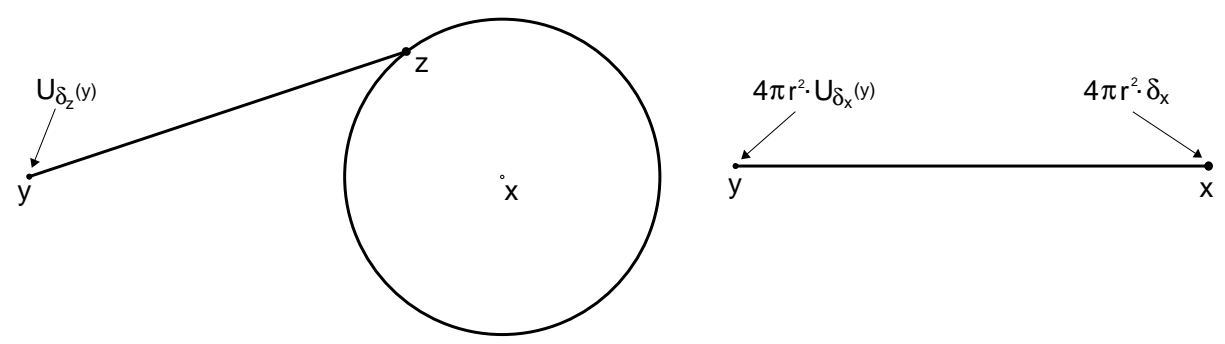

Bild 17 
So speziell dieses Resultat erscheint, ist es doch von allgemeiner Bedeutung in der Potentialtheorie geworden, wie wir gleich sehen werden.

Wenn $\mu$ eine allgemeine Massenverteilung ist, ist das zugehörige Potential $U_{\mu}$ definiert durch Überlagerung der Potentiale $U_{\delta_{y}}$, also durch

$$
U_{\mu}(x)=\int U_{\delta_{y}}(x) \mu(d y)=-\gamma \cdot \int \frac{\mu(d y)}{|x-y|}, \quad x \in \mathbb{R}^{3} .
$$

Wie in (26) ergibt sich die Anziehungskraft einer Massenverteilung $\mu$ aus dem Potential $U_{\mu}$ durch Gradientenbildung; zur Bestimmung der Gravitationskraft kommt es also darauf an, $U_{\mu}$ zu berechnen. Über die Behandlung von Spezialfällen hinaus gelangte man erst im 19. Jahrhundert zu einer allgemeinen Theorie solcher Potentialfunktionen. Gauss entdeckte (vgl. [3], p. 30/31), daß diese Funktionen im massefreien Teil des Raumes die Mittelwerteigenschaft besitzen. Wenn $\mu$ ein endliches positives Maß auf $\mathbb{R}^{3}$ ist, etwa mit kompaktem Träger supp $\mu$, bezeichne man den massefreien Teil des Raumes mit $D=\mathbb{R}^{3} \backslash \operatorname{supp} \mu$. Der Satz von Gauss besagt dann, daß für alle $x \in D$ und für alle $r>0$ mit $\left\{z \in \mathbb{R}^{3}:|z-x| \leq r\right\} \subset D$ gilt

$$
U_{\mu}(x)=\frac{1}{4 \pi r^{2}} \int_{S_{r}(x)} \sigma(d z) U_{\mu}(z) .
$$

Im massefreien Teil des Raumes ist also der Wert von $U_{\mu}$ an einer Stelle $x$ gleich dem Mittelwert von $U_{\mu}$, genommen über die Sphäre $S_{r}(x)$.

Diese Eigenschaft von Potentialfunktionen ist äquivalent zu Newtons Resultat für die Kugel. Um das zu sehen, genügt es, (29) zu spezialisieren auf $\mu=\delta_{y}$ (aus diesem Spezialfall kann man (29) wieder zurückgewinnen, indem man nach dem Maß $\mu(d y)$ integriert), also

$$
U_{\delta_{y}}(x)=\frac{1}{4 \pi r^{2}} \int_{S_{r}(x)} \sigma(d z) U_{\delta_{y}}(z), \quad|x-y|>r,
$$

zu betrachten. In welcher Beziehung stehen die Formeln (30) und (27) zueinander? Sie gehen auseinander hervor, wenn man eine Vertauschung der Variablen gemäß

$$
U_{\delta_{a}}(b)=U_{\delta_{b}}(a), \quad a, b \in \mathbb{R}^{3},
$$

vornimmt (aufgrund von (25) ist die Gültigkeit von (31) klar). Hinter der formalen Vertauschbarkeitsbeziehung (31) steckt ein allgemeines Symmetrieprinzip, demzufolge Ursache (Punktmasse) und Wirkung (Probepunkt) miteinander vertauschbar sind. Anschaulich heißt das, daß man die Mittelwerteigenschaft des Potentials $U_{\delta_{y}}$ "sehen" kann, indem man in Bild 17 eine Umdeutung vornimmt und den Probepunkt $y$ als diejenige Punktmasse betrachtet, um deren Potential es geht.

Die tatsächliche Tragweite der Mittelwerteigenschaft ist erst zu Beginn unseres Jahrhunderts klar geworden, als Koebe gezeigt hat, daß diese Eigenschaft charakteristisch ist für harmonische Funktionen. 
Bemerkung. Der Verfasser bedankt sich bei Felix Friedrich für die Anfertigung der Zeichnungen und bei R. Reibold und der Redaktion der Elemente der Mathematik für wertvolle Hinweise, die zu einer Verbesserung des Manuskripts beigetragen haben.

\section{Literatur}

[1] Archimedes: Werke. Im Anhang (p. 382-423): Des Archimedes Methodenlehre von den mechanischen Lehrsätzen. Wissenschaftliche Buchgesellschaft, Darmstadt 1983.

[2] Chandrasekhar, S.: Newton's Principia for the Common Reader. Clarendon Press. Oxford 1995.

[3] Gauss, C.F.: Allgemeine Lehrsätze in Beziehung auf die im verkehrten Verhältnis des Quadrats der Entfernung wirkenden Anziehungs- und Abstoßungskräfte. Ostwalds Klassiker Nr. 2 (ed. A. Wangerin), Leipzig 1912.

[4] Littlewood, J.E.: A Mathematician's Miscellany. Methuen \& Co., London 1953.

[5] Newton, I.: Mathematical Principles of Natural Philosophy (ed. F. Cajori), 3rd ed., University of California Press 1946.

[6] Pólya, G.: Mathematik und plausibles Schließen, Band 1. Birkhäuser, Basel 1962.

[7] Toeplitz, O.: Die Entwicklung der Infinitesimalrechnung. Wissenschaftliche Buchgesellschaft. Darmstadt 1972.

[8] van der Waerden, B.L.: Einfall und Überlegung in der Mathematik. Elemente der Mathematik 8 (1953), 121-129.

[9] van der Waerden, B.L.: Erwachende Wissenschaft. Birkhäuser, Basel 1966.

\section{Reinhard Lang}

Institut für Angewandte Mathematik

Universität Heidelberg

Im Neuenheimer Feld 294

D-69120 Heidelberg 